\title{
Continuum random phase approximation method applied to the inclusive transverse electron scattering response
}

\author{
E. Bauer \\ Departamento de Física, Facultad de Ciencias Exactas, Universidad Nacional de La Plata, La Plata, 1900, Argentina \\ A. Ramos and A. Polls \\ Departament d'Estructura i Constituents de la Matèria, Universitat de Barcelona, Diagonal 647, E-08028, Spain
}

(Received 28 May 1996)

\begin{abstract}
A continuum random phase approximation (RPA) method for evaluating the structure function of nuclear matter is developed, where exchange terms for any particle-hole interaction are explicitly included. The method is applied to the inclusive transverse quasielastic electron scattering response at momentum transfer ranging from $q=300$ to $550 \mathrm{MeV} / c$. The interaction employed is a $g^{\prime}$ Landau-Migdal constant plus a $(\pi+\rho)$-meson exchange interaction. A comparison with the standard ring series is made showing that the inclusion of finite range effects in the exchange terms is necessary. [S0556-2813(96)01312-X]
\end{abstract}

PACS number(s): 25.30.Fj, 21.60.Jz, 24.10.-i

\section{INTRODUCTION}

Correlations of the random phase approximation (RPA) type are relevant in many nuclear physics problems, ranging from low to medium energy processes. These correlations are well established at low energy [1]. Going to the continuum, such as in the quasielastic peak region for electron scattering, there are some technical complications. In this region, RPA correlations are important at low momentum transfer but the role played by exchange terms for a general particle-hole $(p h)$ interaction is still not clear.

The more elemental approach to this problem relied on the ring approximation using effective Landau-Migdal parameters to account for the exchange terms in an approximate way. This is equivalent to assuming that the exchange contribution of the $p h$ interaction is of zero range. There are several schemes to calculate the RPA response with a more general interaction in the continuum. One is the doorway state expansion method of Brieva et al. [2]. The method was applied to the longitudinal response function and the photoabsorption on ${ }^{12} \mathrm{C}$. Alternatively, Shigehara et al. [3] developed a method based on matrix inversion. Using this formalism they calculated the spin longitudinal, spin transverse, and longitudinal responses which were compared to the ring results. Also Buballa et al. [4] developed a formalism based on a Sturmian function expansion for continuum RPA with a general interaction. They applied the formalism to both quasielastic longitudinal and transverse response functions for ${ }^{12} \mathrm{C}$. Finally, García Recio et al. [5] developed a selfconsistent RPA with the inclusion of exchange terms but limited to a Skyrme-type interaction. They applied the formalism to nuclear matter and liquid ${ }^{3} \mathrm{He}$.

In this paper, we develop a simple method which accounts for RPA correlations in nuclear matter for any interaction including the exchange terms. The method can be used to study both the longitudinal and transverse nuclear responses for inclusive quasielastic electron scattering. However, we will focus on the study of the transverse response using the standard Landau-Migdal parameter $g^{\prime}$ plus a $(\pi+\rho)$-meson exchange as a model interaction. The election of the transverse channel with this interaction is due to two reasons. First, it allows for a comparison with the more complex work of Ref. [4]. Second, even if the method is general, it is particularly suitable for this channel as will be made clear later in this work.

The contribution of direct $p h$ terms to the RPA response for a general interaction can be summed up to infinite order (ring series). Unfortunately, this is not possible for exchange terms. In this work we present a scheme to account for such terms in a simple way and analyze their magnitude. More specifically, we present the formalism in Sec. II, where the residual interaction is divided into a pure contact term and a piece containing a contact term plus a finite range interaction. As will be shown there, the separation is made by demanding that the second piece of the interaction gives rise to a fast convergence of the RPA series, which can then be treated up to second order. The remaining pure contact interaction allows a summation up to infinite order. In Sec. III, we present some numerical results, showing that exchange terms are relevant, and make comparison with other methods. Finally, the main conclusions are summarized in Sec. IV.

\section{FORMALISM}

Let us first introduce the transverse $(T)$ structure function per unit volume

$$
S_{T}(\mathbf{q}, \hbar \omega)=-\frac{\mathbf{1}}{\pi \Omega} \operatorname{Im}\left\langle 0\left|\mathcal{O}_{T}^{\dagger} G(\hbar \omega) \mathcal{O}_{T}\right| 0\right\rangle
$$

where $\Omega$ is the volume. The structure function is related to the response function, $R_{T}(\mathbf{q}, \hbar \omega)$, through the usual dipole electromagnetic form factor, $G_{E}(q, \hbar \omega)$. In Eq. (1), $\hbar \omega$ represents the excitation energy and $\mathbf{q}$ the three-momentum transfer. The operator $\mathcal{O}_{T}$ is the external excitation operator which is given by 


$$
\begin{aligned}
\mathcal{O}_{T}= & \frac{1}{2 m q} \sum_{i=1}^{A}\left(\frac{1+\tau_{3}(i)}{2}\left[\mathbf{q} \times\left\{\mathbf{p}_{i}^{\prime}, e^{i \mathbf{q} \cdot \mathbf{x}_{i}}\right\}\right]\right. \\
& \left.+i \frac{\mu_{s}+\mu_{v} \tau_{3}(i)}{2}\{\mathbf{q} \times[\boldsymbol{\sigma}(i) \times \mathbf{q}]\} e^{i \mathbf{q} \cdot \mathbf{x}_{i}}\right),
\end{aligned}
$$

where $m$ is the nucleonic mass and $\mathbf{x}_{i}$ and $\mathbf{p}_{i}^{\prime}$ denote the intrinsic coordinates and momentum operators for individual nucleons. The values of $\mu_{s}$ and $\mu_{v}$, which are related to the proton and neutron magnetic moments, are $\mu_{s}=0.88$ and $\mu_{v}=4.70$.

The nuclear ground state is denoted by $|0\rangle$ while the polarization propagator $G(\hbar \omega)$ is given by

$$
G(\hbar \omega)=\frac{1}{\hbar \omega-H+i \eta}-\frac{1}{\hbar \omega+H-i \eta}
$$

where $H$ is the nuclear Hamiltonian. As usual, $H$ is separated into a one-body part, $H_{0}$, and a residual interaction, $V$, given by

$$
\begin{aligned}
V(l)= & \frac{f_{\pi}^{2}}{\mu_{\pi}^{2}} \Gamma_{\pi}^{2}(l)\left(g \boldsymbol{\sigma} \cdot \boldsymbol{\sigma}^{\prime}+\widetilde{g}^{\prime}(l) \boldsymbol{\tau} \cdot \boldsymbol{\tau}^{\prime} \boldsymbol{\sigma} \cdot \boldsymbol{\sigma}^{\prime}\right. \\
& \left.+\widetilde{h}^{\prime}(l) \boldsymbol{\tau} \cdot \boldsymbol{\tau}^{\prime} \boldsymbol{\sigma} \cdot \hat{\boldsymbol{l}} \boldsymbol{\sigma}^{\prime} \cdot \boldsymbol{l}\right)
\end{aligned}
$$

with

$$
\begin{gathered}
\widetilde{g}^{\prime}(l)=g^{\prime}-\frac{\Gamma_{\rho}^{2}(l)}{\Gamma_{\pi}^{2}(l)} C_{\rho} \frac{l^{2}}{l^{2}+\mu_{\rho}^{2}}, \\
\widetilde{h^{\prime}}(l)=-\frac{l^{2}}{l^{2}+\mu_{\pi}^{2}}+\frac{\Gamma_{\rho}^{2}(l)}{\Gamma_{\pi}^{2}(l)} C_{\rho} \frac{l^{2}}{l^{2}+\mu_{\rho}^{2}},
\end{gathered}
$$

where $\mu_{\pi} \hbar c\left(\mu_{\rho} \hbar c\right)$ is the pion (rho) rest mass, $C_{\rho}=2.18$ and the Landau-Migdal parameters $g$ and $g^{\prime}$ account for short range correlations. For the form factor of the $\pi N N$ $(\rho N N)$ vertex we have taken

$$
\Gamma_{\pi, \rho}(l)=\frac{\Lambda_{\pi, \rho}^{2}-\left(\mu_{\pi, \rho} \hbar c\right)^{2}}{\Lambda_{\pi, \rho}^{2}+(\hbar c l)^{2}}
$$

with $\Lambda_{\pi}=1.3 \mathrm{GeV}$ and $\Lambda_{\rho}=2 \mathrm{GeV}$. The static limit to the $(\pi+\rho)$-meson exchange interaction has been taken in Eq. (4). An energy dependent interaction makes minor changes in the result.

The Hartree-Fock ground state, $|\mathrm{HF}\rangle$, was adopted as a model for our ground state and the set of intermediate states has been restricted to one-particle-one-hole (1p1h) excitations. Under these assumptions, the structure function $S_{T}(\mathbf{q}, \hbar \omega)$, can be redrawn as

$$
\begin{aligned}
S_{T}(\mathbf{q}, \hbar \omega)= & -\frac{1}{\pi \Omega} \operatorname{Im} \sum_{\substack{1 \mathrm{p} 1 \mathrm{~h} \\
1 \mathrm{p}^{\prime} 1 \mathrm{~h}^{\prime}}}\left\langle\mathrm{HF}\left|\mathcal{O}_{T}^{\dagger}\right| 1 \mathrm{p} 1 \mathrm{~h}\right\rangle \\
& \times\left\langle 1 \mathrm{p} 1 \mathrm{~h}|G(\hbar \omega)| 1 \mathrm{p}^{\prime} 1 \mathrm{~h}^{\prime}\right\rangle\left\langle 1 \mathrm{p}^{\prime} 1 \mathrm{~h}^{\prime}\left|\mathcal{O}_{T}\right| \mathrm{HF}\right\rangle
\end{aligned}
$$

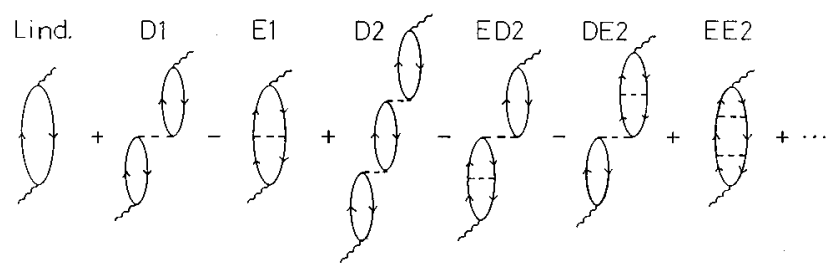

FIG. 1. Goldstone diagrams stemming from the insertion of Eq. (10) into Eq. (8). In every diagram two wavy lines represent the external probe with momentum and energy $(\mathbf{q}, \hbar \omega)$. Only forwardgoing contributions are shown. Diagram Lind represents the Lindhard function. Diagrams $D 1$ and $E 1$ are the direct and exchange first order contribution to the RPA response, respectively, while diagrams $D 2, E D 2, D E 2$, and $E E 2$ are the second-order contributions.

The presence of $V$ makes $G(\hbar \omega)$ nondiagonal in the particle-hole basis. To treat this, the standard Dyson equation is employed:

$$
G(\hbar \omega)=G^{0}(\hbar \omega)+G^{0}(\hbar \omega) V G(\hbar \omega),
$$

where $G^{0}(\hbar \omega)$ results from replacing the total Hamiltonian $H$ in Eq. (3) by the one-body part, $H_{0}$. The matrix elements for the first perturbative terms of Eq. (9) are given by

$$
\begin{aligned}
\left\langle 1 \mathrm{p} 1 \mathrm{~h}|G(\hbar \omega)| 1 \mathrm{p}^{\prime} 1 \mathrm{~h}^{\prime}\right\rangle= & \left\langle 1 \mathrm{p} 1 \mathrm{~h}\left|G^{0}(\hbar \omega)\right| 1 \mathrm{p} 1 \mathrm{~h}\right\rangle \delta_{p p^{\prime}} \delta_{h h^{\prime}} \\
& +\left\langle 1 \mathrm{p} 1 \mathrm{~h}\left|G^{0}(\hbar \omega)\right| 1 \mathrm{p} 1 \mathrm{~h}\right\rangle \\
& \times\left\langle 1 \mathrm{p} 1 \mathrm{~h}|V| 1 \mathrm{p}^{\prime} 1 \mathrm{~h}^{\prime}\right\rangle_{a} \\
& \times\left\langle 1 \mathrm{p}^{\prime} 1 \mathrm{~h}^{\prime}\left|G^{0}(\hbar \omega)\right| 1 \mathrm{p}^{\prime} 1 \mathrm{~h}^{\prime}\right\rangle \\
& +\cdots
\end{aligned}
$$

where $\left\langle 1 \mathrm{p} 1 \mathrm{~h}|V| 1 \mathrm{p}^{\prime} 1 \mathrm{~h}^{\prime}\right\rangle_{a}$ stands for antisymmetrized $V$-matrix elements accounting for both direct and exchange terms. The first forwardgoing terms contributing to the structure function are shown in Fig. 1.

If we neglect the exchange part of all the matrix elements or we consider a pure contact interaction, the terms in Eq. (10) can be easily summed up to infinite order leading to the usual ring approximation. In Fig. 1, this sum is represented by the diagrams labeled Lind, $D 1, D 2, \ldots$, where the nuclear interaction is limited to destroy and create a particlehole pair.

To obtain the antisymmetric structure function in the RPA scheme, one has to evaluate the exchange terms explicitly. In practice, this can be done up to second order. Higher order terms become a prohibitive calculation for finite range interactions. The question then is if the first three terms of Eq. (10) can account for the full sum. To see this, let us compare the infinite ring series (i.e., direct terms) with the sum of direct terms up to second order. For numerical purposes we will restrict ourselves to a $g^{\prime}$ plus a $(\pi+\rho)$-meson exchange interaction. In Fig. 2, we make this comparison for two values of $g^{\prime}$, where the free structure function $S_{T}{ }^{0}$ (diagram labeled Lind in Fig. 1) was subtracted. We see that for $g^{\prime}=0.5$ the accordance is almost exact. This accordance is not good for $g^{\prime}=0.95$. While, as shown in the literature [3], a $g^{\prime} \approx 0.5-0.7$ is taken to partially account for the exchange terms, a value $g^{\prime} \approx 0.7-0.95$ should be employed if the ex- 


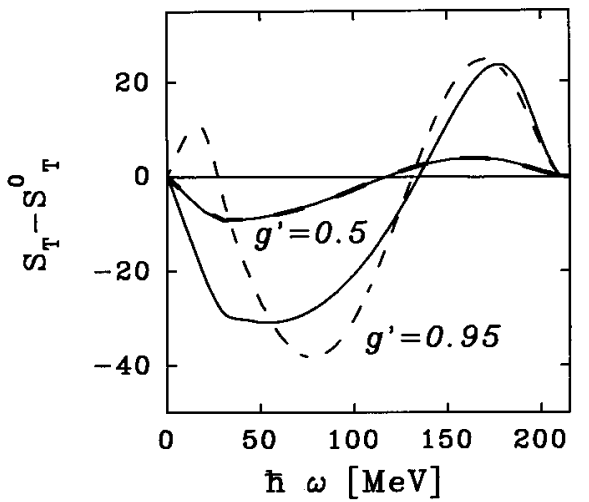

FIG. 2. Comparison between the ring series (full lines) and the sum of terms up to second order (dashed lines) for $g^{\prime}=0.5$ and $g^{\prime}=0.95$ where the free structure function was subtracted. Graphically both results coincide for the $g^{\prime}$ value 0.5 .

change terms are to be evaluated explicitly. In a separate publication [6], we perform an extensive study of the influence of the exchange terms on the nuclear response, including other types of diagrams than those discussed in this paper. Our study, therefore, requires the use of a $g^{\prime} \approx 0.7-0.95$, which has been shown in Fig. 2 not to reproduce the ring series when the sum is restricted up to second order. Consequently, there is no reason to believe that an explicit evaluation of the exchange diagrams of $p h$ type up to second order (see diagrams $E 1, E D 2, D E 2$, and $E E 2$ in Fig. 1) would provide, when added to the infinite ring series, a good representation of the antisymmetric RPA response. However, going to infinite order in the exchange terms is a difficult task. Below we present an easy prescription that allows one to obtain a reliable approximation of the exact RPA response without having to calculate exchange diagrams beyond second order in the finite range interaction explicitly.

As we have mentioned above, for a pure contact interaction it is possible to sum the fully antisymmetric series of Eq. (10). Taking this into account, together with the fact that, for some values of $g^{\prime}$, the ring (direct) series can be reproduced by its terms up to second order in the residual interaction, we divide the interaction as follows:

$$
V=V_{1}+V_{2}
$$

where $V_{1}$ is a pure contact interaction $\left(V_{1}=g_{1}^{\prime}\right)$ and $V_{2}$ contains a contact $\left(g_{2}^{\prime}\right)$ plus the exchange of the $(\pi+\rho)$ mesons. The $V_{2}$ interaction is chosen to ensure a fast convergence of the ring series which, as will be shown later, also implies a fast convergence of Eq. (10), allowing terms of third and higher order in $V_{2}$ to be neglected.

The polarization propagator of Eq. (10) can now be drawn as

$$
G(\hbar \omega)=G_{1}(\hbar \omega)+G_{2}(\hbar \omega)+G_{12}(\hbar \omega),
$$

where
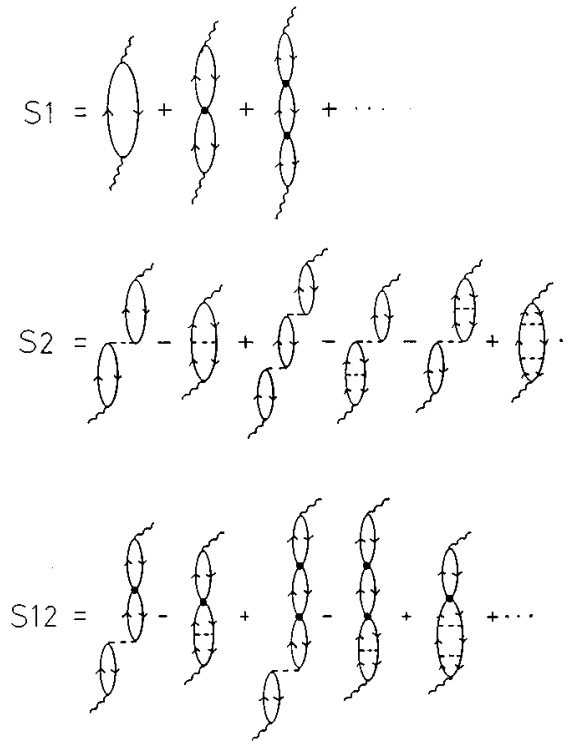

FIG. 3. Goldstone diagrams stemming from Eqs. (13)-(15). In every diagram a dashed line represents the nuclear interaction $V_{2}$ which is a general nonlocal interaction, while a dot between particle-hole bubbles represents the pure contact interaction $V_{1}$. Line $S 1$ is the usual ring series for $V_{1}$. Line $S 2$ is the sum of firstand second-order RPA-type diagrams for the $V_{2}$ interaction, where exchange matrix elements are explicitly included. Finally, line $S 12$ displays some interference terms between $V_{1}$ and $V_{2}$. In all cases, the $V_{2}$ interaction is included up to second order.

$$
\begin{aligned}
G_{1}(\hbar \omega)= & G^{0}(\hbar \omega)+G^{0}(\hbar \omega) V_{1} G^{0}(\hbar \omega) \\
+ & G^{0}(\hbar \omega) V_{1} G^{0}(\hbar \omega) V_{1} G^{0}(\hbar \omega)+\cdots \\
G_{2}(\hbar \omega)= & G^{0}(\hbar \omega) V_{2} G^{0}(\hbar \omega) \\
& +G^{0}(\hbar \omega) V_{2} G^{0}(\hbar \omega) V_{2} G^{0}(\hbar \omega), \\
G_{12}(\hbar \omega)= & G^{0}(\hbar \omega) V_{1} G^{0}(\hbar \omega) V_{2} G^{0}(\hbar \omega) \\
& +G^{0}(\hbar \omega) V_{2} G^{0}(\hbar \omega) V_{1} G^{0}(\hbar \omega) \\
& +G^{0}(\hbar \omega) V_{1} G^{0}(\hbar \omega) V_{1} G^{0}(\hbar \omega) V_{2} G^{0}(\hbar \omega) \\
& +\cdots
\end{aligned}
$$

Inserting Eq. (12) into Eq. (8) one can define three different contributions to the structure function, $S_{1}, S_{2}$, and $S_{12}$, associated to $G_{1}, G_{2}$, and $G_{12}$, respectively. In the case of $S_{1}$, since $V_{1}$ is a pure contact interaction, the direct and exchange terms are equivalent and they can both be summed up to infinite order by evaluating the ring series of Eq. (13) with redefined values for the Landau-Migdal parameters entering in the interaction. In the case of $S_{2}$, Eq. (14) is only considered up to second order, while for $S_{12}$, Eq. (15) is also evaluated up to infinite order in the $V_{1}$ interaction keeping terms up to first order in $V_{2}$ as it will be justified below. In Fig. 3, a graphical representation of this division is shown.

\section{RESULTS AND DISCUSSION}

As an application of this method, the structure function of nonrelativistic nuclear matter at a Fermi momentum $k_{F}=1.36 \mathrm{fm}^{-1}$ is analyzed in detail for a momentum transfer 
TABLE I. RPA contribution to the structure function in nuclear matter at $k_{F}=1.36 \mathrm{fm}^{-1}$ for a momentum transfer $q=410 \mathrm{MeV} / c$. The energy is given in $\mathrm{MeV}$, while the structure function is in units of $\left(10^{-5} \mathrm{MeV}^{-1} \mathrm{fm}^{-3}\right)$. The notation for $S_{1}$, $S_{2}$, and $S_{12}$ is the same as in Fig. 3, while that for $D 1, E 1, D 2$, and $E 2$ is the same as in Fig. 1, where $E 2$ is the sum of all second-order exchange contributions $E D 2, D E 2$, and $E E 2$. Note that the free structure is included in $S_{1}$. Column $S_{T}$ collects the sum of all contributions.

\begin{tabular}{|c|c|c|c|c|c|c|c|}
\hline \multirow[t]{2}{*}{$\hbar \omega$} & \multirow[t]{2}{*}{$S_{1}$} & \multicolumn{4}{|l|}{$S_{2}$} & \multirow[t]{2}{*}{$S_{12}$} & \multirow[t]{2}{*}{$S_{T}$} \\
\hline & & $D 1$ & $E 1$ & $D 2$ & $E 2$ & & \\
\hline 40 & 24.903 & -10.396 & 4.035 & 1.333 & -0.503 & -0.374 & 18.997 \\
\hline 80 & 39.923 & -5.466 & 2.178 & -0.295 & 0.186 & 0.780 & 37.308 \\
\hline 120 & 51.820 & 0.801 & 0.050 & -0.561 & 0.338 & 1.065 & 53.511 \\
\hline 160 & 50.682 & 3.697 & -1.552 & 0.096 & -0.040 & -0.826 & 52.056 \\
\hline
\end{tabular}

$q=410 \mathrm{MeV} / c$. We have employed a $g^{\prime}=0.95$, taken from Ref. [3]. The separation of the interaction is performed by considering $g_{1}^{\prime}=0.45$ as the only contribution for the contact interaction $V_{1}$, while $V_{2}$ contains $g_{2}^{\prime}=0.5$ plus the $(\pi+\rho)$-meson exchange. As $V_{1}$ is a pure contact force it can be redefined to account for antisymmetrization. This is shown in full detail in Ref. [3]. For completeness, we outline here the procedure in our particular case. The direct piece of the $p h$ interaction,

$$
V_{1}=\frac{f_{\pi}^{2}}{\mu_{\pi}^{2}} g_{1}^{\prime} \boldsymbol{\tau} \cdot \boldsymbol{\tau}^{\prime} \boldsymbol{\sigma} \cdot \boldsymbol{\sigma}^{\prime}
$$

contains only a $g_{1}^{\prime}$ term. The total (direct plus exchange) $p h$ interaction is given by

$$
\left(V_{1}\right)_{\mathrm{ant}}=V_{1}+\left(V_{1}\right)_{\mathrm{exch}},
$$

where the exchange piece is constructed via the spin-isospin exchange operator $P_{\sigma} P_{\tau}$

$$
\begin{aligned}
\left(V_{1}\right)_{\mathrm{exch}}= & -P_{\sigma} P_{\tau} V_{1} \\
= & \frac{f_{\pi}^{2}}{\mu_{\pi}^{2}}\left(f_{1, E}+f_{1, E}^{\prime} \boldsymbol{\tau} \cdot \boldsymbol{\tau}^{\prime}+g_{1, E} \boldsymbol{\sigma} \cdot \boldsymbol{\sigma}^{\prime}\right. \\
& \left.+g_{1, E}^{\prime} \boldsymbol{\tau} \cdot \boldsymbol{\tau}^{\prime} \boldsymbol{\sigma} \cdot \boldsymbol{\sigma}^{\prime}\right),
\end{aligned}
$$

and contains contributions in all the spin-isospin channels, with $f_{1, E}=-\frac{9}{4} g_{1}^{\prime}, f_{1, E}^{\prime}=\frac{3}{4} g_{1}^{\prime} g_{1, E}=\frac{3}{4} g_{1}^{\prime}$, and $g_{1, E}^{\prime}=-\frac{1}{4} g_{1}^{\prime}$. Only $g_{1}$ and $g_{1}^{\prime}$ are relevant in the transverse structure function. Following Eq. (17), their antisymmetrized values are $\left(g_{1}\right)_{\text {ant }}=g_{1, E}=0.3375$ and $\left(g_{1}^{\prime}\right)_{\text {ant }}=g_{1}^{\prime}+g_{1, E}^{\prime}=0.3375$. Using the redefined interaction $\left(V_{1}\right)_{\text {ant }}$, the ring series accounts for both direct and exchange terms up to infinite order and builds up the $S_{1}$ contribution to the structure function.

In Fig. 2 we showed that the sum of direct terms up to second order in $V_{2}$ equalled the ring series for that interaction. By restricting the sum of Eq. (14) to the second-order terms, we have implicitly assumed that the convergence is also valid for the exchange terms, a fact which is not evident as the exchange terms have different momentum dependence. The assumption is based on the results shown in

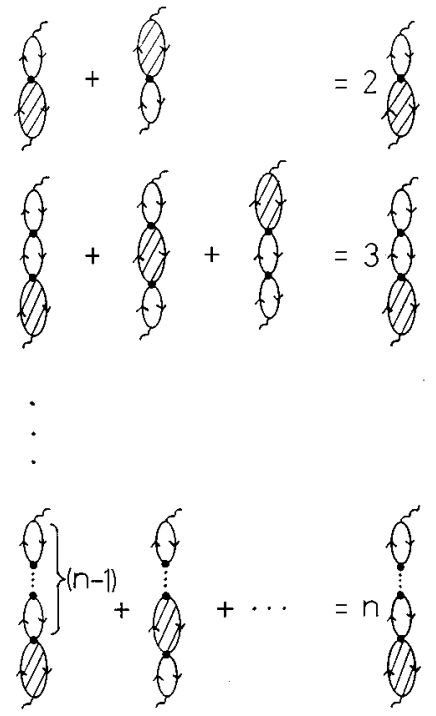

FIG. 4. Goldstone diagrams used to evaluate $S 12$. The shaded bubble represents the sum of direct and exchange contributions of $V_{2}$ (see explanation in the text).

Table I, where the different contributions to the total structure function $S_{T}$ are shown. By comparing columns $E 1$ with $D 1$ and $E 2$ with $D 2$, it is observed that exchange terms are smaller than the corresponding direct ones. Since the higher order direct terms will be negligible small, it is plausible to believe that, according to the trend of the results shown in Table I, the same will hold true for the exchange terms.

Explicit expressions for the different diagrams are shown in the Appendix. The multiple integrations have been performed using a Monte Carlo technique. The numerical evaluation of the exchange graph EE2 in Fig. 1 is particularly difficult and cannot be given with good accuracy. However, this lack of accuracy does not affect the whole calculation as its value is very small. As a consequence, some higher order contributions, as the one shown at the end of line $S_{12}$ in Fig. 3 , can also be neglected. In Fig. 4 we present all diagrams used to evaluate $S_{12}$, where the shaded bubble represents the sum of direct $(D 1)$ and exchange $(E 1)$ contributions of $V_{2}$. The first graph on the top LHS of Fig. 4 is the sum of the first two graphs shown in Fig. 3 for $S_{12}$. Similarly, the first graph in the second line of Fig. 4 is the sum of the third and fourth graphs of $S_{12}$ in Fig. 3. All the other diagrams on the LHS of Fig. 4 represent additional contributions to $S_{12}$, present in Eq. (15), which have not been drawn in Fig. 3. These contributions are obtained by allowing the shaded bubble to be located in all different positions in the diagrams. All contributions yield identical results and they can be accounted for by the factor appearing on the RHS of Fig. 4 . The resulting $S_{12}$ is the sum of all graphs on the RHS of the equations shown in Fig. 4 with their corresponding factors. All graphs are first order in $V_{2}$. The summation up to infinite order in $V_{1}$ gives a modified ring series as can be seen in Eq. (A6) of the Appendix. ${ }^{1}$ The possibility of summing this series up to infinite order, together with the fact that the

\footnotetext{
${ }^{1}$ In the Appendix we show the $V_{2}$ exchange contribution only. It is straighforward to obtain the direct one.
} 
TABLE II. Comparison between the ring approximation and the total RPA contribution to the structure function of nuclear matter at $k_{F}=1.36 \mathrm{fm}^{-1}$, for three different transferred momenta: $q=300$, 410 , and $550 \mathrm{MeV} / c$. Units are the same as in Table I. A $g^{\prime}$ plus a $(\pi+\rho)$-meson exchange interaction was used to calculate the ring series, taking $g^{\prime}=0.55$ for $\overline{S^{\text {ring1 }}}$ and $g^{\prime}=0.7$ for $\overline{S^{\text {ring2 }}}$. The results

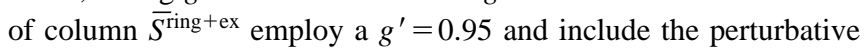
exchange graphs up to second order in the interaction. The results of our method are given in column $S^{\mathrm{RPA}}$, where the employed value of $g_{2}^{\prime}$ is explicitly shown. The free structure function was subtracted for convenience as indicated in the text.

\begin{tabular}{|c|c|c|c|c|}
\hline $\begin{array}{l}q=300 \mathrm{MeV} / c \\
\hbar \omega\end{array}$ & $\bar{S}^{\text {ring1 }}$ & $\bar{S}^{\text {ring2 }}$ & $\bar{S}^{\text {ring }+ \text { ex }}$ & $\overrightarrow{S^{\mathrm{RPA}}}\left(g_{2}^{\prime}=0.30\right)$ \\
\hline 30 & -19.698 & -23.434 & -36.762 & -29.143 \\
\hline 60 & -13.867 & -19.842 & -12.322 & -22.463 \\
\hline 90 & 4.154 & 1.549 & -6.674 & 2.295 \\
\hline 120 & 10.442 & 18.578 & 25.908 & 17.711 \\
\hline $\begin{array}{l}q=410 \mathrm{MeV} / c \\
\hbar \omega\end{array}$ & $\bar{S}^{\text {ring1 }}$ & $\bar{S}^{\text {ring2 }}$ & $\bar{S}^{\text {ring+ex }}$ & $\bar{S}^{\mathrm{RPA}}\left(g_{2}^{\prime}=0.50\right)$ \\
\hline 30 & -13.028 & -21.063 & -29.544 & -29.461 \\
\hline 60 & -11.415 & -20.344 & -22.089 & -27.626 \\
\hline 90 & -6.571 & -13.755 & -14.402 & -17.378 \\
\hline 120 & -0.079 & -2.533 & -5.662 & -1.833 \\
\hline 150 & 4.959 & 8.810 & 6.617 & 11.843 \\
\hline 180 & 4.854 & 10.816 & 16.724 & 12.733 \\
\hline $\begin{array}{l}q=550 \mathrm{MeV} / c \\
\hbar \omega\end{array}$ & $\bar{S}^{\text {ring1 }}$ & $\bar{S}^{\text {ring2 }}$ & $\bar{S}^{\text {ring }+ \text { ex }}$ & $S^{\mathrm{RPA}}\left(g_{2}^{\prime}=0.85\right)$ \\
\hline 30 & 4.794 & -1.588 & -4.523 & -5.475 \\
\hline 60 & 7.849 & -2.706 & -7.463 & -9.160 \\
\hline 90 & 8.120 & -2.973 & -8.086 & -9.636 \\
\hline 120 & 6.294 & -2.500 & -6.917 & -7.480 \\
\hline 150 & 3.333 & -1.514 & -4.384 & -3.867 \\
\hline 180 & 0.195 & -0.313 & -1.138 & 0.089 \\
\hline 210 & -2.303 & 0.772 & 1.868 & 3.169 \\
\hline 240 & -3.563 & 1.420 & 3.908 & 4.324 \\
\hline 270 & -3.262 & 1.392 & 3.995 & 3.436 \\
\hline
\end{tabular}

second-order contributions in $V_{2}$ are negligible for $S_{12}$, makes the division of the interaction useful for practical purposes.

In Table II, we present the structure function calculated in several approximations for three values of the momentum transfer. The free structure function, $S_{T}^{0}$, has been substracted in order to emphasize the differences between the approximations. This is indicated by the notation $\bar{S}_{T}=S_{T}-S_{T}^{0}$. The results of our method (last column, labeled $S^{\mathrm{RPA}}$ ) are compared with those obtained summing the direct ring series employing an interaction $V=g^{\prime}+(\pi+\rho)$ with $g^{\prime}=0.55\left(\bar{S}^{\text {ring1 }}\right)$ and $g^{\prime}=0.70\left(\bar{S}^{\text {ring2 }}\right)$, as suggested in Ref. [3]. These values of $g^{\prime}$ result from an average procedure over the exchange matrix elements on the Fermi surface. The first value does not include a screening effect contained in the second one (see Ref. [3]). Note that, in the evaluation of $\bar{S}$ RPA , different values of $g_{2}^{\prime}$ have been employed for different values of the momentum transfer $q$, according to the prescription of a fast convergence to the ring series. The resulting $g_{2}^{\prime}$ values are $0.3,0.5$, and 0.85 for $q=300,410$, and $550 \mathrm{MeV} / c$, respectively. In Table II we observe that the ring approximation underestimates the RPA correlations, especially in the case $\overline{S^{\text {ring1 }}}$. This is due to an overestimation of the exchange contribution stemming from the average procedure for $g^{\prime}$. However, even if there is a manifest difference between our results and the ring approximation, this difference gets smaller as $q$ tends to zero, that is, when one goes closer to the Landau limit. The reason is that it is precisely in this low momentum transfer region where the effective $g^{\prime}$ value to account for exchange terms was derived. In the case of $q=550 \mathrm{MeV} / c$, the results of the ring approximation with $g^{\prime}=0.55\left(\bar{S}^{\text {ring1 }}\right)$ even have a different sign than the RPA results, although it must be noted that RPA correlations are smaller in this region of momentum transfer. The results of Table II show that, in the intermediate momentum transfer region, the average procedure is not adequate, while RPA correlations are still significant. Note that in this region all correlations both in the ground state and in the final state are relevant [7].

Our method must be viewed as a convenient way of adding the RPA series up to infinite order in which only diagrams up to second order in a piece of the interaction $\left[V_{2}=g_{2}^{\prime}+(\pi+\rho)\right]$ need to be explicitly calculated. For the method to be useful, one must therefore check how it compares with the technically similar but conceptually more straightforward alternative procedure consisting in calculating the direct ring series with the complete interaction $\left[V=g^{\prime}+(\pi+\rho)\right]$ and the exchange graphs (E1, ED2, $D E 2$, and $E E 2$ in Fig. 1) only up to second order in $V$. Column $\overline{S^{\text {ring+ex }}}$ in Table II shows that the convergence of exchange terms in the alternative procedure is not fast in general and depends on the $g^{\prime}$ value employed. Observe that the discrepancy between the $\bar{S}^{\text {ring+ex }}$ value and our result $\overline{S^{\mathrm{RPA}}}$, gets smaller as $g_{2}$ approaches to $g^{\prime}$, which is when both procedures are equivalent.

We want to stress that the difference between the $\bar{S}^{\text {ring }+ \text { ex }}$ approximation and ours, relays on higher order exchange diagrams which we are including. To clarify this point, let us consider a third-order exchange diagram in the total interaction $V$. In $\bar{S}^{\text {ring+ex }}$ this diagram is not considered at all. However, it is partially included in our scheme in the way we describe in the following. First of all, this diagram is split into several contributions stemming from the division of $V$ into $V_{1}$ and $V_{2}$. The contribution which contains only $V_{1}$ is part of $S_{1}$; contributions which only contain $V_{2}$ or are second order in $V_{2}$ (see the last diagram of line $S_{12}$ in Fig. 3) are neglected. Finally, contributions that are first order in $V_{2}$ are included in $S_{12}$. Note that we have numerically checked that the second order in $V_{2}$ exchange diagram (diagram EE2 in Fig. 1) gives a small contribution, which suggests that neglecting second- and higher order $V_{2}$ diagrams is a reliable assumption as already discussed in relation to Table I.

The total response function, $R_{T}(\mathbf{q}, \hbar \omega)$, related to $S_{T}$ through the dipole electromagnetic form factor, is shown in Fig. 5 for several values of momentum transfer. Our results are represented by the full line, the free response by the long dashed line, and $\bar{S}^{\text {ring+ex }}$ by the short dashed line. It is observed that the RPA correlations shift strength from the low to the high energy region, keeping the energy-weighted sum rule unchanged. For $q=300 \mathrm{MeV} / c$, we observe a non- 


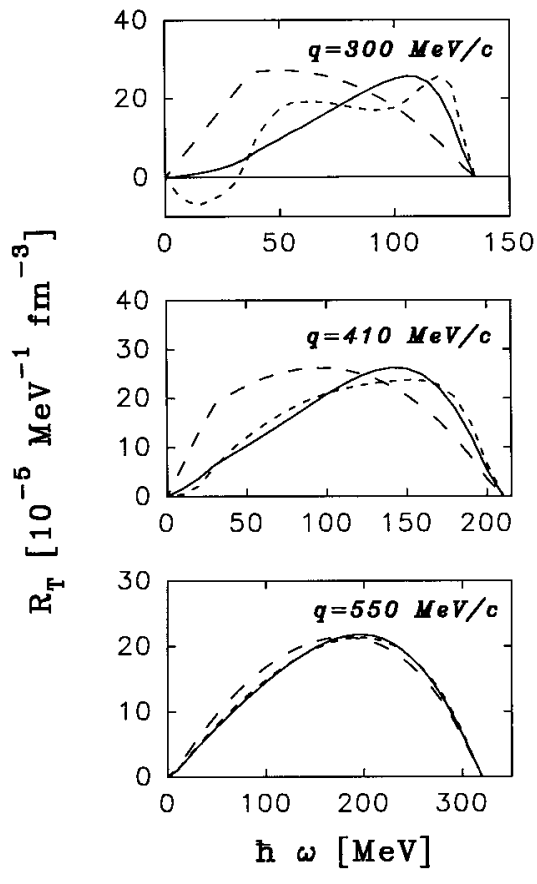

FIG. 5. Transverse response function at several values of the momentum transfer as a function of the energy $\hbar \omega$. The free response function (long-dashed line), the response for $\overline{S^{\text {ring+ex }}}$ (shortdashed line) and our result (full line) are shown.

physical negative value for $\bar{S}^{\text {ring+ex }}$ at low energy. This is a simple consequence of having neglected higher order exchange diagrams. Note that this pathology is absent in our method. Our results show a qualitative agreement with those of Fig. 6 in Ref. [4]. The comparison of the effect of the exchange terms cannot be made deeper because the nucleus considered in Ref. [4], ${ }^{12} \mathrm{C}$, is too light for a nuclear matter description. As other mechanisms beyond RPA correlations, like higher order correlations [7-15] or meson exchange currents (MEC) [16,17], are important in the transverse channel, a comparison with data is not possible. A more complete version, where the exchange contribution for higher order correlations are included, is in progress [6]. However, we have focused on RPA correlations because they deserve special attention.

Finally, it is important to note that our method can be applied to a more general interaction with all the LandauMigdal terms, as these additional constant terms can be properly antisymmetrized up to infinite order in the ring series. In particular, the method can be easily applied to the longitudinal channel. In this case, the Landau-Migdal parameters $f$ and $f^{\prime}$ should be added to the residual interaction as they dominate the direct contribution. Still, it is the $g^{\prime}$ parameter which should be tuned up to apply the method.

\section{CONCLUSION}

We have presented a simple scheme to evaluate the full antisymmetric RPA series contributing to the structure function in nuclear matter. The prescription is based on two ingredients. The first one is the separation of the interaction in a pure contact term plus a contribution which guarantees a fast convergence to the ring series. The second is the fact that for a pure contact interaction it is possible to sum the full antisymmetric RPA series up to infinite order. The result is that explicit evaluation of the exchange contribution is important and cannot be reduced to the evaluation of the ring series with the standard average prescription for $g^{\prime}$, especially for intermediate values of the momentum transfer. This stresses also the importance of the inclusion of the pion (in particular its tensor force) which is not present in the ring approximation.

\section{ACKNOWLEDGMENTS}

We are grateful to A. de Pace for valuable comments. This work was partially supported by the Spanish Research Council, DGICYT, under Contract No. PB92-0761. E.B. acknowledges support from the Catalan Research Council, CIRIT. He is also grateful for the warm hospitality extended to him while visiting the University of Barcelona. E. Bauer is a fellow of the Consejo Nacional de Investigaciones Científicas y Técnicas, Conicet.

\section{APPENDIX}

In this appendix, we present explicit expressions for the different exchange graphs needed to build up our antisymmetric RPA for nuclear matter. We do not reproduce here the ring series as it can be found in many references (see, for example, Ref. [18]).

Even if in our numerical example we have focused on a $g^{\prime}$ plus a $(\pi+\rho)$-meson exchange interaction, we prefer to include also the parameter $g$ of Eq. (4), for completeness.

In the following, we use dimensionless quantities $\mathbf{Q}=\mathbf{q} / \mathbf{k}_{\mathbf{F}}$ and $\nu=\hbar \omega / 2 \varepsilon_{F}$, where $k_{F}$ and $\varepsilon_{F}$ are the Fermi momentum and Fermi energy, respectively.

Graph $E 1$ of Fig. 1:

$$
\begin{aligned}
{\left[S_{E 1}(\mathbf{Q}, \nu)\right]_{T}=} & -\frac{1}{(2 \pi)^{5}}\left(\frac{f_{\pi}^{2}}{4 \pi \hbar c}\right) \frac{k_{F}{ }^{4}}{\hbar c \mu_{\pi}^{2}} \int d^{3} h \int d^{3} k \theta(1-h) \theta(|\mathbf{h}+\mathbf{Q}|-\mathbf{1}) \theta(\mathbf{1}-|\mathbf{h}+\mathbf{k}|) \theta(|\mathbf{h}+\mathbf{k}+\mathbf{Q}|-\mathbf{1}) \Gamma_{\pi}^{2}(k) \\
& \times\left\{\left[3\left(g_{2}+\widetilde{g_{2}}\right)+\widetilde{h^{\prime}}\right] 4[\mathbf{h}(\mathbf{h}+\mathbf{k})-(\mathbf{Q} \cdot \mathbf{h}) \mathbf{Q} \cdot(\mathbf{h}+\mathbf{k})] / \mathbf{Q}^{2}\right]+\left(\mu_{s}{ }^{2}+\mu_{v}{ }^{2}\right) Q^{2}\left(-g_{2}\right)+\left(-3 \mu_{s}{ }^{2}+\mu_{v}{ }^{2}\right) \\
& \times Q^{2}\left[\widetilde{g_{2}}+\widetilde{h^{\prime}}(\hat{\mathbf{k}} \cdot \hat{\mathbf{Q}})^{2}\right\}\left(-\frac{1}{\pi} \operatorname{Im}\right)\left[\left(\frac{1}{2 \nu-\left(Q^{2}+2 \mathbf{h} \cdot \mathbf{Q}\right)+i \eta}-\frac{1}{2 \nu+\left(Q^{2}+2 \mathbf{h} \cdot \mathbf{Q}\right)}\right)\right. \\
& \left.\times\left(\frac{1}{2 \nu-\left[Q^{2}+2(\mathbf{h}+\mathbf{k}) \mathbf{Q}\right)+i \eta}-\frac{1}{2 \nu+\left[Q^{2}+2(\mathbf{h}+\mathbf{k}) \mathbf{Q}\right)}\right)\right],
\end{aligned}
$$

where we have defined 
Graph EE2 of Fig. 1:

$$
\widetilde{g}_{2}(k)=g_{2}^{\prime}-\frac{\Gamma_{\rho}^{2}(k)}{\Gamma_{\pi}^{2}(k)} C_{\rho} \frac{k^{2}}{k^{2}+\mu_{\rho}^{2}} .
$$

$$
\begin{aligned}
& {\left[S_{E E 2}(\mathbf{Q}, \nu)\right]_{\mathbf{T}}=\frac{1}{(2 \pi)^{7}}\left(\frac{f_{\pi}^{2}}{4 \pi \hbar c}\right)^{2} \frac{4 m c^{2} k_{F}^{5}}{\left(\hbar c \mu_{\pi}^{2}\right)^{2}} \int d^{3} h \int d^{3} k \int d^{3} k^{\prime} \theta(|\mathbf{h}+\mathbf{Q}|-\mathbf{1})} \\
& \times \theta(1-|\mathbf{h}|) \theta(\mathbf{1}-|\mathbf{h}+\mathbf{k}|) \theta(|\mathbf{h}+\mathbf{k}+\mathbf{Q}|-\mathbf{1}) \theta\left(\mathbf{1}-\left|\mathbf{h}+\mathbf{k}+\mathbf{k}^{\prime}\right|\right) \theta\left(\left|\mathbf{h}+\mathbf{k}+\mathbf{k}^{\prime}+\mathbf{Q}\right|-\mathbf{1}\right) \Gamma_{\pi}^{2}(k) \Gamma_{\pi}^{2}\left(k^{\prime}\right) \\
& \times\left(\left\{5\left[9\left(\widetilde{g_{2}}\right)^{2}+\widetilde{h^{\prime}} 2+6 \widetilde{g_{2}} \widetilde{h^{\prime}}\right]+9\left(g_{2}\right)^{2}+9 g_{2} \widetilde{g_{2}}+6 g_{2} \widetilde{h^{\prime}}\right\} 8\left[\mathbf{h}\left(\mathbf{h}+\mathbf{k}+\mathbf{k}^{\prime}\right)-(\mathbf{Q} \cdot \mathbf{h}) \mathbf{Q} \cdot\left(\mathbf{h}+\mathbf{k}+\mathbf{k}^{\prime}\right) / \mathbf{Q}^{2}\right]\right. \\
& +\frac{3 \mu_{s}{ }^{2}-\mu_{v}{ }^{2}}{2}\left\{Q^{2}\left(g_{2}\right)^{2}+g_{2} \widetilde{g}_{2}+g_{2} \widetilde{h^{\prime}}\left[(\mathbf{Q} \cdot \hat{\mathbf{k}})^{2}+\left(\mathbf{Q} \cdot \hat{\mathbf{k}}^{\prime}\right)^{2}\right]\right\}+\frac{\mu_{s}{ }^{2}+\mu_{v}{ }^{2}}{2} Q^{2}\left(g_{2}\right)^{2}+\frac{9 \mu_{s}{ }^{2}+\mu_{v}{ }^{2}}{2} \\
& \times\left(Q^{2}\left\{\left(\widetilde{g_{2}}\right)^{2}+\widetilde{h^{\prime 2}}\left[2\left(\hat{\mathbf{k}} \cdot \widehat{k^{\prime}}\right)^{2}-\mathbf{1}\right]\right\}+\widetilde{h^{\prime 2}}\left[(\mathbf{Q} \cdot \hat{\mathbf{k}})^{2}+\left(\mathbf{Q} \hat{\mathbf{k}}^{\prime}\right)^{2}-\mathbf{2}(\mathbf{Q} \cdot \hat{\mathbf{k}})\left(\mathbf{Q} \cdot \hat{\mathbf{k}}^{\prime}\right)\left(\hat{\mathbf{k}} \cdot \hat{\mathbf{k}}^{\prime}\right)\right]\right. \\
& \left.+\widetilde{g_{2}} \widetilde{h^{\prime}}\left[(\mathbf{Q} \cdot \hat{\mathbf{k}})^{2}+\left(\mathbf{Q} \cdot \hat{\mathbf{k}}^{\prime}\right)^{2}\right]\right)\left(-\frac{1}{\pi} \operatorname{Im}\right)\left[\left(\frac{1}{2 \nu-\left(Q^{2}+2 \mathbf{h} \cdot \mathbf{Q}\right)+i \eta}-\frac{1}{2 \nu+\left[Q^{2}+2 \mathbf{h} \cdot \mathbf{Q}\right]}\right)\right. \\
& \times\left(\frac{1}{2 \nu-\left[Q^{2}+2(\mathbf{h}+\mathbf{k}) \cdot \mathbf{Q}\right]+i \eta} \frac{1}{2 \nu+\left[Q^{2}+2(\mathbf{h}+\mathbf{k}) \mathbf{Q}\right]}\right) \\
& \left.\times\left(\frac{1}{2 \nu-\left[Q^{2}+2\left(\mathbf{h}+\mathbf{k}+\mathbf{k}^{\prime}\right) \mathbf{Q}\right)+i \eta}-\frac{1}{2 \nu+\left[Q^{2}+2\left(\mathbf{h}+\mathbf{k}+\mathbf{k}^{\prime}\right) \mathbf{Q}\right]}\right)\right] .
\end{aligned}
$$

Graph ED2 (DE2) of Fig. 1:

$$
\begin{aligned}
{\left[S_{E D 2}(\mathbf{Q}, \nu)\right]_{\mathbf{T}}=} & -\frac{1}{(2 \pi)^{7}}\left(\frac{f_{\pi}^{5}}{4 \pi \hbar c}\right)^{2} k_{F}^{5} \frac{8 m c^{2}}{\left(\hbar c \mu_{\pi}^{2}\right)^{2}} Q^{2}\left(-\frac{1}{\pi} \operatorname{Im}\right)\left[\mathcal{L}(\mathbf{Q}, \nu) \int d^{3} h \int d^{3} k \theta(1-h) \theta(|\mathbf{h}+\mathbf{Q}|-\mathbf{1}) \theta(\mathbf{1}-|\mathbf{h}+\mathbf{k}|)\right. \\
& \times \theta(|\mathbf{h}+\mathbf{k}+\mathbf{Q}|-\mathbf{1}) \boldsymbol{\Gamma}_{\pi}^{2}(\mathbf{Q}) \boldsymbol{\Gamma}_{\pi}^{2}(\mathbf{k})\left\{\mathbf{4 g}_{2} \mu_{s}^{2}\left[\mathbf{g}_{2}-\widetilde{3}_{2}^{\prime}-\widetilde{3}^{\prime}(\hat{\mathbf{k}} \cdot \hat{\mathbf{Q}})^{2}\right]+4 g_{2}^{\prime} \mu_{v}{ }^{2}\left[-g_{2}+\widetilde{g_{2}}+\widetilde{h^{\prime}}(\hat{\mathbf{k}} \cdot \hat{\mathbf{Q}})^{2}\right]\right\} \\
& \times\left(\frac{1}{2 \nu-\left(Q^{2}+2 \mathbf{h} \cdot \mathbf{Q}\right)+i \eta}-\frac{1}{2 \nu+\left(Q^{2}+2 \mathbf{h} \cdot \mathbf{Q}\right)}\right) \\
& \left.\times\left(\frac{1}{2 \nu-\left[Q^{2}+2(\mathbf{h}+\mathbf{k}) \cdot \mathbf{Q}\right]+i \eta}-\frac{1}{2 \nu+\left[Q^{2}+2(\mathbf{h}+\mathbf{k}) \cdot \mathbf{Q}\right]}\right)\right]
\end{aligned}
$$

with

$$
\mathcal{L}(\mathbf{Q}, \nu)=\int d^{3} p \theta(|\mathbf{p}+\mathbf{Q} / 2|-\mathbf{1}) \theta(\mathbf{1}-|\mathbf{p}-\mathbf{Q} / \mathbf{2}|)\left(\frac{1}{2 \nu-Q^{2}-2 \mathbf{Q} \cdot \mathbf{p}+i \eta}-\frac{1}{2 \nu+Q^{2}+2 \mathbf{Q} \cdot \mathbf{p}}\right) .
$$

Sum of all graphs contributing to $G_{12}$ (see the last line of Fig. 3):

$$
\begin{aligned}
{\left[S_{C 12}(\mathbf{Q}, \nu)\right]_{\mathbf{T}}=} & -\frac{1}{(2 \pi)^{7}}\left(\frac{f_{\pi}^{2}}{4 \pi \hbar c}\right)^{2} k_{F}^{5} \frac{4 m c^{2}}{\left(\hbar c \mu_{\pi}^{2}\right)^{2}} Q^{2}\left(-\frac{1}{\pi} \operatorname{Im}\right)\left[\mathcal{L}(\mathbf{Q}, \nu) \int d^{3} h \int d^{3} k \theta(1-h) \theta(|\mathbf{h}+\mathbf{Q}|-\mathbf{1})\right. \\
& \times \theta(\mathbf{1}-|\mathbf{h}+\mathbf{k}|) \theta(|\mathbf{h}+\mathbf{k}+\mathbf{Q}|-\mathbf{1}) \boldsymbol{\Gamma}_{\pi}^{2}(\mathbf{Q}) \boldsymbol{\Gamma}_{\pi}^{2}(\mathbf{k})\left(4 \frac{g_{1}\left[2-4 g_{1} \mathcal{C}^{\text {int }}(Q) \mathcal{L}(Q, \nu)\right]}{\left[1-4 g_{1} \mathcal{C}^{\text {int }}(Q) \mathcal{L}(Q, \nu)\right]^{2}} \mu_{s}^{2}\left[-g_{2}-3 \widetilde{g_{2}}-3 \widetilde{h^{\prime}}(\hat{\mathbf{k}} \cdot \hat{\mathbf{Q}})^{2}\right]\right. \\
& \left.+4 \frac{\widetilde{g}_{1}^{\prime}\left[2-4 \widetilde{g_{1}^{\prime}} \mathcal{C}^{\text {int }}(Q) \mathcal{L}(Q, \nu)\right]}{\left[1-4 \widetilde{g_{1}} \mathcal{C}^{\text {int }}(Q) \mathcal{L}(Q, \nu)\right]^{2}} \mu_{v}^{2}\left[-g_{2}+\widetilde{g_{2}}+\widetilde{h^{\prime}}(\hat{\mathbf{k}} \cdot \hat{\mathbf{Q}})^{2}\right]\right) \\
& \times\left(\frac{1}{2 \nu-\left(Q^{2}+2 \mathbf{h} \cdot \mathbf{Q}\right)+i \eta}-\frac{1}{2 \nu+\left(Q^{2}+2 \mathbf{h} \cdot \mathbf{Q}\right)}\right) \\
& \left.\times\left(\frac{1}{2 \nu-\left[Q^{2}+2(\mathbf{h}+\mathbf{k}) \cdot \mathbf{Q}\right]+i \eta}-\frac{1}{2 \nu+\left[Q^{2}+2(\mathbf{h}+\mathbf{k}) \mathbf{Q}\right]}\right)\right]
\end{aligned}
$$

where

$$
\mathcal{C}^{\mathrm{int}}(Q)=\left(\frac{f_{\pi}^{2}}{4 \pi \hbar c}\right) \frac{k_{F}}{\pi^{2}} \frac{m c^{2}}{\hbar c \mu_{\pi}^{2}} \Gamma_{\pi}^{2}(Q) .
$$


[1] D. J. Rowe, Nuclear Collective Motion (Methuen, London, 1970).

[2] F. A. Brieva and A. Dellafiore, Phys. Rev. C 36, 899 (1987).

[3] T. Shigehara, K. Shimizu, and A. Arima, Nucl. Phys. A492, 388 (1989).

[4] M. Buballa, S. Drożdż, S. Krewald, and J. Speth, Ann. Phys. 208, 346 (1991).

[5] C. García Recio, J. Navarro, Van Giai Nguyen, and L. L. Salcedo, Ann. Phys. 214, 293 (1992).

[6] E. Bauer, A. Polls, and A. Ramos (unpublished).

[7] E. Bauer, Nucl. Phys. A589, 669 (1995).

[8] S. Drożdż, G. Co', J. Wambach, and J. Speth, Phys. Lett. B 185, 287 (1987).

[9] O. Benhar, A. Fabrocini, and S. Fantoni, Nucl. Phys. A550, 201 (1992).

[10] Y. Horikawa, F. Lenz, and Nimai C. Mukhopadhyay, Phys. Rev. C 22, 1680 (1980).

[11] C. R. Chinn, A. Picklesimer, and J. W. Van Orden, Phys. Rev.
C 40, 790 (1989); 40, 1159 (1989).

[12] F. Capuzzi, C. Giusti, and F. D. Pacati, Nucl. Phys. A524, 681 (1991); F. Capuzzi, ibid. A554, 362 (1993).

[13] P. M. Boucher, B. Castel, and Y. Okuhara, Ann. Phys. 196, 150 (1989).

[14] W. M. Alberico, M. Ericson, and A. Molinari, Ann. Phys. 154, 356 (1984); W. M. Alberico, A. De Pace, A. Drago, and A. Molinari, Riv. Nuovo Cimento 14, 1 (1991).

[15] K. Takayanagi, Phys. Lett. B 230, 11 (1989); Nucl. Phys. A510, 162 (1990); A522, 494 (1991); A522, 523 (1991); A556, 14 (1993).

[16] J. W. Van Order and T. W. Donnelly, Ann. Phys. 131, 451 (1981).

[17] J. E. Amaro, G. Co', A. M. Lallena, Ann. Phys. 221, 306 (1993); Nucl. Phys. A578, 365 (1994).

[18] A. L. Fetter and J. D. Walecka, Quantum Theory of ManyParticle Systems (McGraw-Hill, New York, 1971). 